\title{
Action Potential Modeling in Isolated Rat Hearts During Hypokalemia
}

\author{
Mariano Llamedo ${ }^{1}$, Augusto Santini ${ }^{1}$, Emiliano Diez ${ }^{2}$ \\ ${ }^{1}$ GIBIO, National Technological University, Buenos Aires, Argentina \\ ${ }^{2}$ Institute of Physiology, Medical School, National University of Cuyo, Mendoza, Argentina
}

\begin{abstract}
This study aimed to develop a detector with few physiologically-meaningful parameters, that could be capable of detecting pseudo periodic patterns. The algorithm is based in a signal detection based on a matched filter, and a threshold calculation based on robust stadistics. The evaluation of the detector was performed under a corpus consisting in two sets. One set of human ECGs, and one set of rodent pseudo ECGs. The evaluation was performed with respect to the gold standard annotations, and was calculated in terms of sensitivity $(S)$ and positive predictive value $(P)$. For the human ECG set of recordings, the detector had $100 \mathrm{~S}$ and $99.9 \mathrm{P}$, while for the rodent pseudo ECG set of recordings the results where 97.1 S and 79.1 P. Both sets results are median values. The results obtained under the rodent set where compared to those obtained with another two detectors, where our detector obtained the best results. The algorithm achieved promising results, in a broad set of ECG recordings of very different nature, with the additional capability of further adaptation provided by expert assistance.
\end{abstract}

\section{Introduction}

Malignant arrhythmias are a major cause of morbidity and mortality in the world. Electrolyte imbalance due to potassium deficiency can enhance the risk of arrhythmias. Hypokalemia contributes to reducing survival of cardiac patients and increases the incidence of arrhythmic death. Almost 50\% of patients resuscitated from out-of-hospital ventricular fibrillation have low potassium levels. Furthermore, in advanced stages of chronic kidney disease, sudden cardiac death could be responsible for $60 \%$ of cardiac deaths and is related to the potassium imbalance. In the general population plasma potassium level range from 3.5 to $5.5 \mathrm{mEq} / \mathrm{L}$ and the risk for complications, including arrhythmias, occurs with values below $2.7 \mathrm{mEq} / \mathrm{L}$. However, in other population like in dialyzed chronic kidney disease patients, potassium level lower than $4 \mathrm{mEq} / \mathrm{L}$ is a risk factor for arrhythmic events. It was reported that arrhythmias are more likely to start developing during and after dialy- sis $[1,2]$. During advance chronic kidney disease, potassium slowly accumulates in the intracellular and the extracellular space. During dialysis, potassium can be removed from the body at faster rates producing relative hypokalemia, which could be critical in excitable tissues like the heart. Since we performed experiments in healthy rat hearts, we acutely reduced the potassium level to $1 \mathrm{mEq} / \mathrm{L}$ which match a ratio between extracellular and intracellular space similar to the one described in dialyzed patients that developed arrhythmic episodes [3].

Acute low extracellular potassium exposure triggers arrhythmias due to lengthening in action potential duration, abnormal pacemaker activity, the presence of early and delayed afterdepolarizations and decrease in conduction velocity due to uncoupling of cell-to-cell communication. The latter effect also contributes to the persistence of the reentrant circuits involved in the pathogenesis of arrhythmias. Melatonin protects against several cardiovascular diseases and exhibits antiarrhythmic potential. The antiarrhythmic properties of melatonin have been previously reported mostly in relation to ischemia/reperfusion-induced arrhythmias. The principal mechanism associated with melatonin protection is its well documented antioxidant effect. However, recent reports indicate that melatonin could protect against arrhythmias by other mechanisms like preventing changes in the action potential duration. Furthermore, chronic melatonin treatment in hypertensive rat hearts increased the threshold of ventricular fibrillation induction. This effect was related to up-regulation of myocardial connexin-43, the main protein responsible for electrical coupling. Because of the importance of hypokalemia in cardiac arrhythmogenesis, we investigated whether melatonin will protect against low extracellular potassium induced arrhythmias by preventing action potential lengthening, triggered activity and by preserving gap junction channels function and distribution.

The elevated heart rates developed by rodents, together with the morphological changes induced by changes in the potassium concentration are very challenging for automatic algorithms. In order detect heartbeats and perform measurements in ECG waves and action potential recordings, a semiautomatic approach was used. The objective of 
this work was to develop the a reproducible method for detecting heartbeats and model the action potentials recorded in rats hearts during electrophysiological experimentation, such as the described in this work.

\section{Material and methods}

Isolated rat hearts underwent low potassium perfusion ( $1 \mathrm{mEq} / \mathrm{L})$ in 4 groups: 1) Mel, $100 \mu \mathrm{M}$ melatonin; 2) Luz, luzindole $5 \mu \mathrm{M}$ a melatonin receptor blocker; 3) Mel+Luz melatonin+luzindole; or 4) Control. All procedures were approved by the local Institutional Animal Care and Use Committee which is in agreement with the Guide for Care and Use of Laboratory Animals (National Academy Press, 1996). Rats were housed in metal cages under conditions of controlled temperature and humidity, with food and water ad libitum and exposed to a cycle of 12 hours of light and 12 hours of darkness. In five hearts per group, we registered simultaneously a far field pseudo ECG signal together with the epicardial transmembrane potential using a custom made microelectrode amplifier. Both signals were digitized at a sampling rate of $20 \mathrm{kHz}$ for posterior digital processing and analysis. The analysis consisted in a) detecting heartbeats, b) correcting/validating detections, c) measure parameters in signal averaged AP (SAAP), and finally d) validate time-series AP measurements for posterior statistical analysis.

The detections of heartbeats was performed in the ECG signal with an ad-hoc algorithm described and analyzed in more detail by the same authors in another article presented at this conference. This algorithm perform a twostep detection based on two restrictions: temporal duration of an arbitrary disruptive pattern and minimum time between two consecutive patterns. The algorithm makes a first detection attempt with a matched filter approach and a synthetic pattern for a rough detection, and a final refinement step where the synthetic pattern is replaced with the more promising patterns found. This algorithm provided great automatic performance, and an additional feature: if the algorithm did not provide acceptable results, an expert user can refine the pattern in order to correct detections in a semiautomatic fashion. The details of the algorithm can also be found in an open source implementation $[4,5]$.

The detection of heartbeats allow us to calculate an SAAP by synchronous averaging from all AP's found in a 10 seconds window, enhancing the SNR of the resulting signal [6]. The SAAP was modeled by morphological and temporal features, such as several potential values: the action potential amplitude (APA), the resting membrane (RMP), the action potential duration at a fraction of APA, e.g. at $50 \%$ of APA (APD50) and the maximum rate of depolarization (MRD). Finally, the activation delay between the start of the QRS complex in the signal averaged ECG (SAECG) and the apex of the SAAP was also calculated,

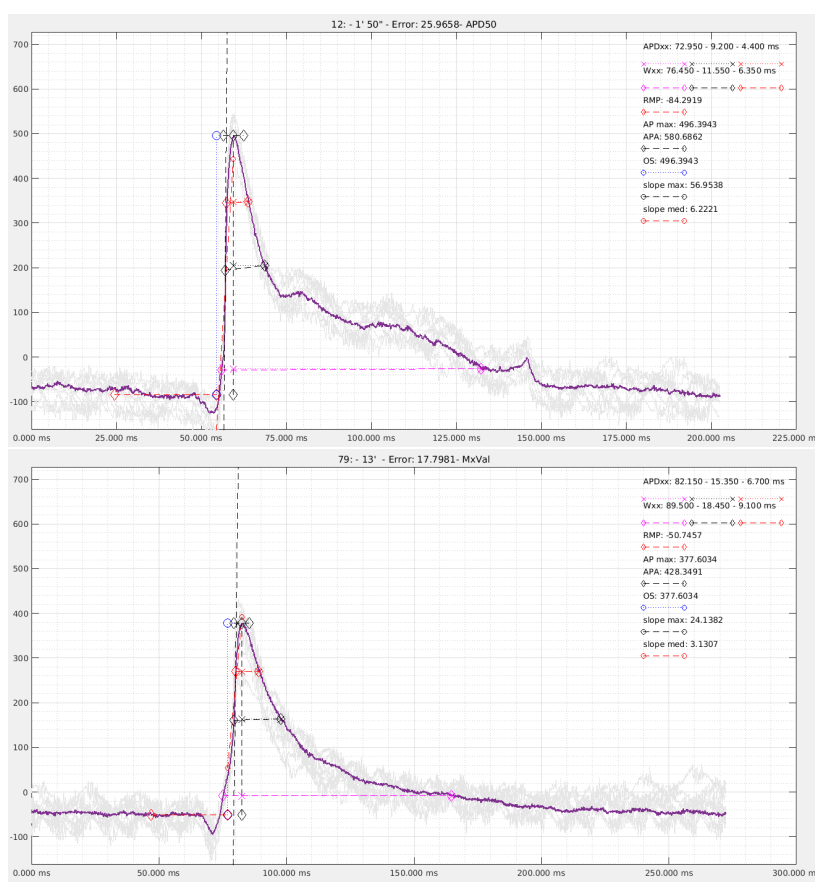

Figure 1. Measurements calculated in an averaged action potential during stabilization (top panel) and hypokalemia (bottom panel).

as a a global estimation of the ventricular conduction velocity. Examples of these measurements are shown in Fig. 1.

The treatments were statistically analyzed by ANOVA for repeated measures, with a confidence level of $\alpha=$ 0.05 .

\section{Results}

Based in the panels in Fig. 3 plus the info from LUZ and MEL+LUZ Action potentials lengthened during hypokalemia and also the fase 0 of the action potentials were delayed in respect to the beginning of the QRS complex but without in the amplitude or the $\mathrm{dV} / \mathrm{dtmax}$ which suggest that $\mathrm{Na}$ channels were not affected.

As expected during hypokalemia, QT interval lengthened in all the groups. All heart developed bradycardia and PR lengthening (in ms: Control $53.2 \pm 5.6$ to $57.4 \pm$ $8.7 *$; Mel $51.7 \pm 4.8$ to $59.4 \pm 6.7 * ;$ Mel+Luz $51.0 \pm$ 4.3 to $58.7 \pm 6.9 *$; and Luz $52.8 \pm 5.7$ to $56.5 \pm 6.4 *$ ). Melatonin was the only treatment that prevented the prolongation in the activation delay induced by hypokalemia (in ms: Control $8.8 \pm 1.9$ to $14.2 \pm 2.0 * * *$; Mel $7.8 \pm 2.4$ to $9.4 \pm 2.8$; Mel+Luz $8.8 \pm 1.3$ to $15.7 \pm 2.2 * * *$; and Luz $8.8 \pm 1.4$ to $13.3 \pm 2.6 *)$. On the other hand, hypokalemia increased QRS duration only the control group (in ms: Control $13.2 \pm 1.7$ to $18.3 \pm 1.7^{* * *}$; Mel $12.7 \pm$ 


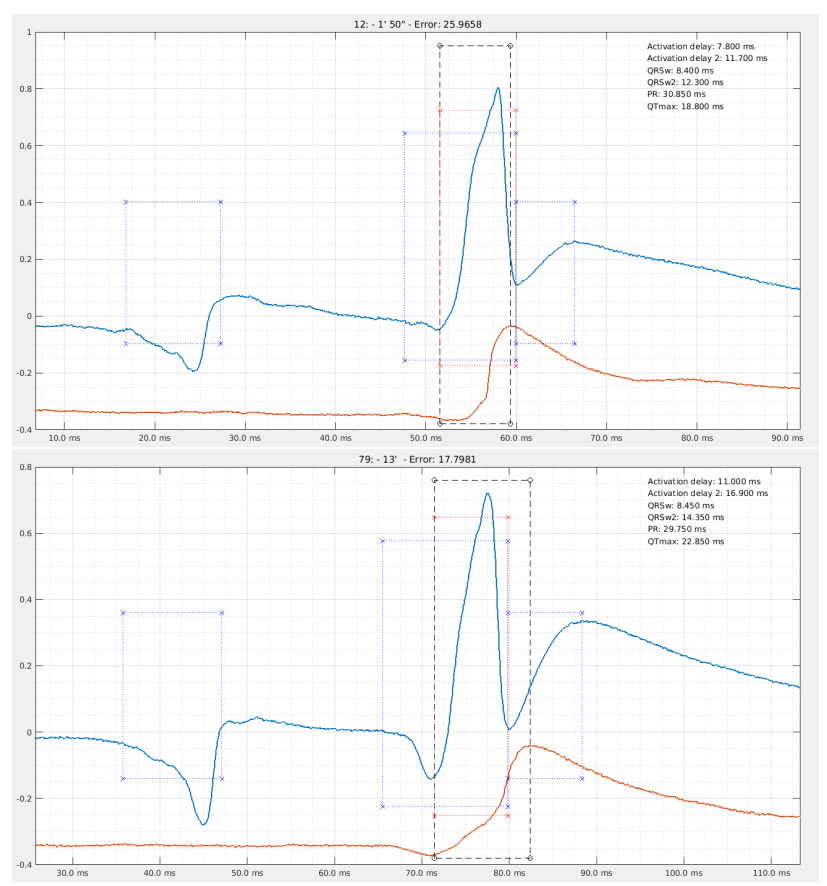

Figure 2. Measurements calculated in both averaged ECG (top-blue) and action potential (bottom-red) during stabilization (top panel) and hypokalemia (bottom panel).

2.6 to $14.4 \pm 2.1$; Mel+Luz $12.1 \pm 2.0$ to $15.2 \pm 3.2$; and Luz $11.2 \pm 1.7$ to $15.4 \pm 1.5)$. $* \mathrm{P}<0.05$, ** $\mathrm{P}<0.01$, and $* * * \mathrm{P}<0.001$ by ANOVA. The proportion of hearts within each group that underwent ventricular fibrillation was only reduced for the melatonin treatment, as is shown in Fig. 4.

\section{Discussion and conclusions}

In this study, we developed a semiautomatic method for detecting and modeling amplitude and intervals among ECG and transmembrane potentials in rat cardiomyocytes. The algorithm is based on an automatic algorithm for detecting arbitrary disruptive patterns, commonly found in cardiovascular signals. The design objective of this method was to increase the analysis capacity of cardiologists without technical knowledge in signal processing and data analysis. This first objective was achieved, however the algorithm has room for improvement, specially in improving the automatic performance. We will address this issue in subsequent works, at the same time that the signal acquisition will be systematized.

The experimentation performed in this work investigated the antiarrhythmic effect of melatonin, and its relation with 1) the action potential morphology and 2) the propagation of the electrical impulses. The control group exhibited an expected behavior with respect to 1), since APD lengthened during hypokalemia (lowK) condition.

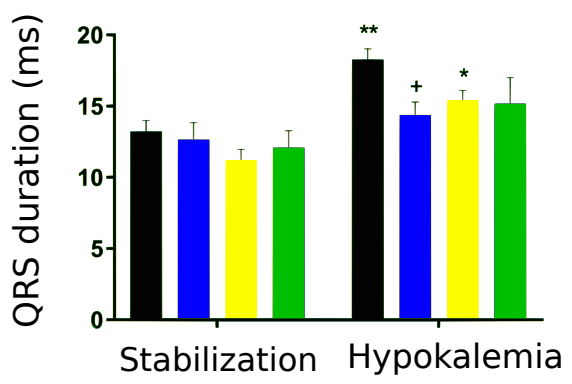

- Control

- Melatonin

Luzindole

- Mel + Luz

Vs. stab.

* $\quad \mathrm{P}<0.05$

$* * \quad \mathrm{P}<0.01$

$* * * \mathrm{P}<0.001$

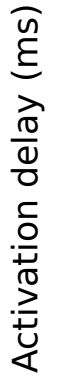

Vs. control

$+\quad \mathrm{P}<0.05$

$++\mathrm{P}<0.01$

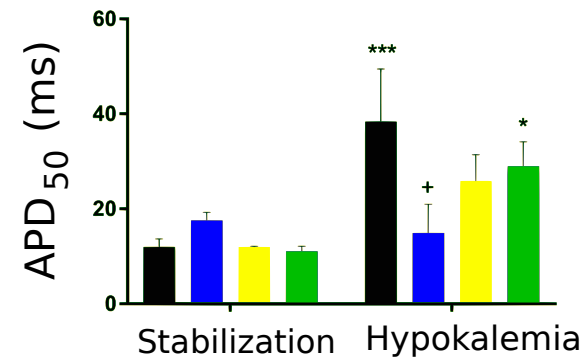

Figure 3. Comparison between stabilization and hypokalemia conditions, and between melatonin and control groups.

Ventricular Fibrillation Incidence (\%)

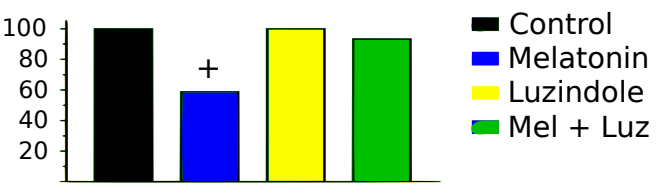

Vs. control $+P<0.05++P<0.01$

Figure 4. Proportion of hearts within each group that underwent ventricular fibrillation.

With respect to the propagation of the electrical impulse, both the action potential delay and the QRS width, surrogates of the conduction velocity through the tissue, also increased during lowK. In contrast, melatonin prevented all indexes to increase, suggesting that the conduction pathway was preserved. As the resting membrane potential was decreased, consequence of the lowK condition, and therefore the sodium current increased, the results may suggest that the pathway is preserved by the connexins or gap junctions. When analyzing the luzindole group, we see a behavior similar than the control group, showing no antiar- 
rhythmic effect at all. However, when luzindole and melatonin are simultaneously used, the net effect of melatonin was reduced as expected (Fig. 3). These results were also confirmed by the proportion of ventricular fibrillations occurred for each group. The melatonin group was the only group with a significant decrease, as shown in Fig. 4.

The results presented in this work suggest that the methodology was useful for analyzing the behavior of electrophysiological signals during experimentation. Further improvements in the automaticity of the methodology will allow further experimentation to contrast the results presented in this work.

\section{Acknowledgments}

This work was supported by projects ASUTNBA0003860, ICUTNBA0004864 and ICUTNBA0004887 from UTN FRBA and J045 from SECTyP-UNCuyo.

\section{References}

[1] Ichikawa H, Nagake Y, Makino H. Signal averaged electrocardiography (SAECG) in patients on hemodialysis. Journal of Medicine 1997;28(3-4):229-243. ISSN 00257850. URL http://www.ncbi.nlm.nih.gov/ pubmed/9355027.

[2] Kovesdy CP, Regidor DL, Mehrotra R, Jing J, McAllister CJ, Greenland S, Kopple JD, Kalantar-Zadeh K. Serum and dialysate potassium concentrations and survival in hemodialysis patients. Clinical Journal of the American Society of Nephrology 2007;2(5):999-1007.

[3] Diez ER, et al. Melatonin, given at the time of reperfusion, prevents ventricular arrhythmias in isolated hearts from fructose fed rats and spontaneously hypertensive rats. Journal of Pineal Research ;55(2):166-173.

[4] Demski AJ, Llamedo M. ecg-kit a Matlab Toolbox for Cardiovascular Signal Processing. Journal of Open Research Software 2016;4(1):2-5.

[5] Llamedo M. ecg-kit: A matlab toolbox for cardiovascular signal processing, 5 2015. URL http: / / marianux. github.io/ecg-kit/.

[6] Sörnmo L, Laguna P. Bioelectrical Signal Processing in Cardiac and Neurological Applications. Elsevier, 2005. ISBN 0-12-437552-9.

Address for correspondence:

Mariano Llamedo, llamedom@ frba.utn.edu.ar

Medrano 951 (C1179AAQ) C.A.B.A., Argentina 